\title{
Nonlinear Structured-Illumination Microscopy with Photoswitchable Molecules
}

\author{
E.H. Rego ${ }^{a, b}$ and M.G.L. Gustafsson ${ }^{b}$ \\ ${ }^{a}$ Graduate Group in Biophysics, University of California, San Francisco, CA 94158 \\ ${ }^{\mathrm{b}}$ Howard Hughes Medical Institute, Janelia Farm Research Center, Ashburn, VA 20147;
}

The resolution of a widefield light microscope can be extended beyond the classical diffraction limit using nonuniform illumination [1]. In the case of a one-dimensional sinusoidally varying pattern, the resolution is increased by an amount equal to the inverse line-spacing $\mathbf{k}_{\mathbf{1}}$ of the illumination pattern. The set of spatial frequencies that can be generated are limited in the same way as the set of spatial frequencies that can be detected; therefore, the resolution improvement is at most a factor of two compared to conventional microscopy.

Dramatically greater resolution can be achieved if the response of the sample is made to be nonlinear with the incoming illumination intensity. The data will contain higher order harmonics that are multiples of $\mathbf{k}_{\mathbf{1}}$, and correspondingly greater resolution can be obtained using these high frequency components. This idea has been previously explored in a widefield microscope by saturating the excited state of a fluorophore [2]. Unfortunately, saturation requires extremely high light intensities that can accelerate photobleaching, and damage even fixed cells. Photoswitching has been theorized for many years to be a good candidate for resolution enhancement because of the genetically encoded photo-switchable molecules available, and the low light intensity required to switch the molecules. This idea has been implemented on purified protein adsorbed to non-biological structures, in either a one-dimensional widefield configuration $[3,4]$ or in a point-scanning configuration [5], each analyzed with real-space methods.s

Here, we use the photoswitchable fluorescent protein Dronpa to generate a nonlinear response from the sample using low light intensities. We detect information in two dimensions at greater than four times the conventional diffraction limit with a widefield TIRF structured illumination setup. The raw data is analyzed with frequency-space based methods, which allow for detection of illumination patterns with a spatial frequency close to the conventional resolution limit itself. To verify the enhanced resolution we look at purified microtubules coated with Dronpa, and the actin cytoskeleton in fixed mammalian cells.

\section{References}

[1] M.G.L. Gustafsson, J. Microsc. 198 (2000).

[2] M.G.L. Gustafsson, Proc. Natl. Acad. Sci. USA (2005).

[3] M. Heilemann et al., Angew. Chem. Int. Ed. 47 (2008).

[4] M. Hofmann et al., Proc. Natl. Acad. Sci. USA (2005).

[5] P. Dedecker et al., ,. Am. Chem. Soc. Deddck 